\title{
Design A New Approach In Mobile Ad - Hoc Network Routing With Bandwidth Efficient Protocol
}

\author{
Pallavi Kaura ${ }^{1}$, Er. Harshit ${ }^{2}$ \\ ${ }^{I}$ (Mtech Student, ECE Department, DIET, Kharar, Mohali) \\ ${ }^{2}$ (HOD and AP, ECE Department, DIET, Kharar, Mohali)
}

\begin{abstract}
Ad hoc wireless networks consist of mobile nodes that communicate with each other without an infrastructure. A reduction in routing overload and efficient use of resources are two very important issues in these networks. In this paper, a new routing algorithm called POSITION BASED HYBRID ROUTING ALGORITHM (PBHRA)with BANDWIDTH. The main goal is effective use of static bandwidth by reducing the routing overload but also battery life, density, cost and delay time are efficiently used by reducing the amount of data to be held and the number of operations to be done for routing by any node in the network. In order to achieve above goals, the principle of DYNAMIC SOURCE ROUTING (DSR) protocol has been utilized.
\end{abstract}

Keyworkds: - Ad-Hoc, Routing, Wireless Routing, MATLAB

\section{INTRODUCTION}

Wireless networks have been quite popular since they appeared in 1970. The popularity of wireless networks arises from supplying data access opportunity to the users anywhere. The technological tendency of users is to communicate with wireless and mobile devices. The wide spread usage of cellular phones, portable computers and palmtop computers with WLAN is the greatest indicator of this.

Wireless networks can be classified into two categories: with infrastructure and without infrastructure networks. Wireless networks with infrastructure, also known as cellular networks, have permanent bases stations, which are used to connect each other through links. Mobile nodes communicate with each other as through these base stations.

Wireless networks without infrastructure also known as MANET as composed of random moving mobile nodes without central controls such as a predefined infrastructure or base station. Now a days, these mobile nodes that can take place on airports, ships, trucks, automobiles and people in very small devices are widely used in many industrial and commercial applications. The usage areas given above make mobility of the nodes compulsory.

MANETs have many characteristics: they do not have central control, all nodes have wireless interface, frequent topology changes as a result of freely moving of nodes, nodes have limited resources (like bandwidth and battery life), they have physical security risk more than wired algorithms and there are inadequate simetric links. Also, each mobile node has to make the routing processes which are performed in wired network router because routing process in wireless networks is made by transmitting from node to node.

These characteristics of MANETs must be considered while developing new algorithms. In addition, the overload of routing algorithm must be minimized in order to efficiently consume insufficient sources.

The process of finding shortest path is usually realized by using protocols based on distance vector or link state routing algorithms. These algorithms do not give good performance in MANET that has limited bandwidth and does not have a central control structure. For this reason, changes on indicated protocols must be made or new protocols must be developed in the routing process in wireless networks. Therefore, in this study, a new routing algorithm working on position information of the nodes was proposed by considering the characteristics of wireless ad hoc networks.

\section{ROUTING ALGORITHMS IN AD - HOC NETWORKS}

There are many routing algorithms developed for wireless ad hoc networks in the literature. These algorithms are classified into three main groups as table driven, on demand and hybrid algorithms.

\section{- Table - Driven Routing Algorithms:-}

Table driven routing algorithms are also called proactive algorithms. Protocols that use this algorithms find all paths between source - destination pairs in a network and form the newest path with periodic route updates. Updates messages are sent even if there no topological changes. The protocols which are in this category are developed by changing distance vector and link state algorithms. These protocols store routing 
information in routing tables and give result very slowly because of periodic updates of tables. This working strategy is not very suitable for wireless ad hoc networks because of a great deal of routing overload.

\section{DESTINATION SEQUENCED DISTANCE VECTOR:-}

It is commonly used algorithm by means of its performance criteria among table driven protocols category. DSDV protocol adds a sequence number to the routing information protocol's category. DSDV protocol adds a sequence number field is used to differentiate between old and new routes. Each node maintains a routing table which next hop information for all reachable destinations. The routing table is updated by periodic advertisements or whenever new information is available.

The performance of protocol is mainly dependent on interval value of sending of periodic updates. If this interval is very short, a big amount of routing overload will occur. If the interval is long, delay will appear in receiving the most updated information. If there are many moving nodes in the network, This protocol will not be efficient.

\section{- On - Demand Routing Algorithms:-}

Unlike table driven algorithms, on demand routing algorithms do not form route information routing information among nodes. Routes are founded only in case of necessity. Routes are formed only when needed, in other words when any of the nodes wants to send a packet. Therefore routing overload is less than table driven algorithms. However, packet delivery fraction is low because every node does not keep updated route information.

\section{DYNAMIC SOURCE ROUTING (DSR):-}

In this algorithm, sender node determines the entire route of sent packets and adds the determined route information to the header of packet. This process can be made as static or dynamic. DSR protocol uses dynamic source routing.

DSR algorithm does not send periodic updates. However, there is routing overload because all route information is added into each data packet. This overload increases in state of mobility and traffic density.

\section{AD HOC ON DEMAND DISTANCE VECTOR (AODV):-}

According to this algorithm, each node keeps routing table, but opposite of DSDV, which is a table driven algorithm, it does not have to keep routes to all other nodes. Like DSR algorithm, route determining process is made via broadcasts.

AODV finds multi routes among source and destination pairs. The situation avoids overload of a new route determination pairs. This situation avoids overload of a new route determination process if there is a break path in a route. In addition, it allows user to select and control route for load balancing and similar operations. Route cache is very useful in state of low mobility. Nevertheless, in the case of high mobility, overload occurs.

\section{- Hybrid Routing Algorithms:-}

Hybrid routing algorithms aim to use advantages of table driven and on demand algorithms and minimize their disadvantages. Position Based Routing Algorithms that is classified in the hybrid routing algorithms category include the properties of table driven and on demand protocols and are usually interested in localized nodes. Localization is realized by GPS that is used to determined geographical positions of nodes.

Position changes which occur because of nodes mobility in MANETs cause changes in routing tables of nodes. The GPSs, which are embedded in nodes, are used to update information in tables in position - based algorithms different from the table driven and on demand algorithm.

The GPSs have become preferred systems as they provide latitude, longitude and height values at high reliability and low cost. Some of the GPS based hybrid routing algorithms are: Directional Routing Algorithm (DIR), Most forward within radius (MFR), geographical distance routing (GEDIR) and Distance routing effect algorithm for mobility (DREAM).

\section{POSITIONED BASED HYBRID ROUTING ALGORITHM}

The proposed algorithm not only aims to efficiently use the bandwidth by reducing the routing overhead but also battery life, cost, delay time are efficiently used by reducing the amount of data to be held and the number of operations to be done for routing by any node in network.

Hybrid routing algorithms include the features of table driven and on demand protocols and localized nodes. Localization means the determination of geographical position of nodes by means of Global Position System (GPS) or other mechanism. GPSs have been preferred systems as they provide latitude, longitude and altitude with low cost and high accuracy. In this type of protocols, geographical position of the current node and 
other nodes are effective while making decision of routing. Recent availability of small inexpensive low power GPS receivers and techniques provided justification for applying positioned based routing methods.

Position based routing algorithm that is classified in the hybrid algorithms category include the properties of table driven and on demand protocols and are usually interested in localized nodes. Localization is realized by GPS that is used to determine geographical positions of nodes.

Position changes which occur because of nodes mobility in MANET cause change in routing of nodes, are used to update information in tables in position - based algorithms different from the table driven and on demand algorithms.

A reduction in routing overload and efficient use of static bandwidth are two very important issues in these networks. In the work, a new routing algorithm called Position Based Hybrid Routing Algorithm (PBHRA) with Static Bandwidth developed to optimize bandwidth usage of ad hoc networks.

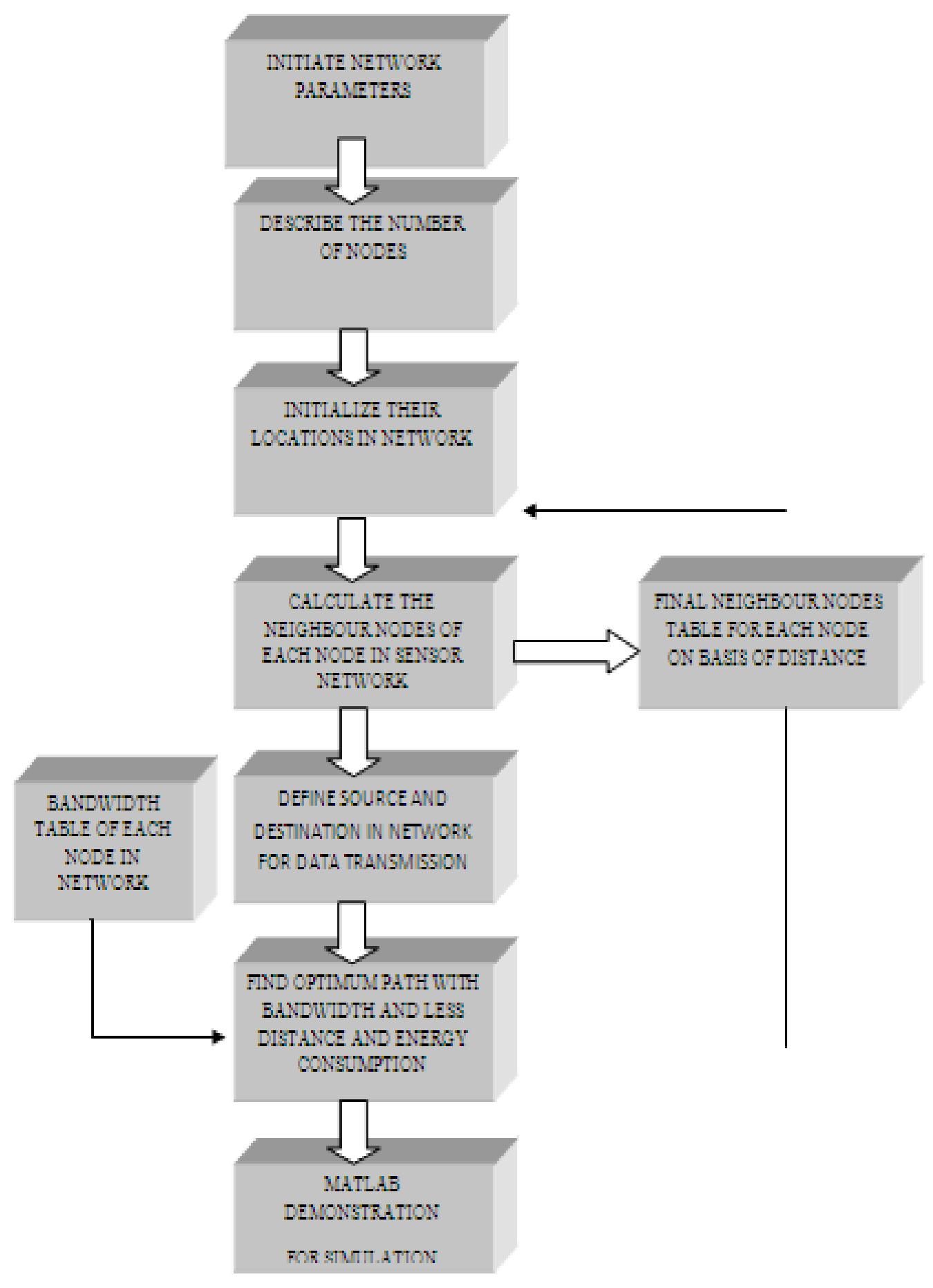




\section{SIMULATION AND RESULTS}

- The very first step is to initiate the parameters of the networks such as

1) How much area is covered under the network

2) Location of nodes

3) Numbers of nodes etc.

- Detecting total number of nodes in the network area by connecting a sensor to each of them. We have presented an algorithm to detect the compromised nodes in wireless sensor networks. These nodes are capable of performing tasks some processing, gathering sensory information and communicating with other connected nodes in the network.

- Now these nodes are initialized, which means to provide initial values before communication according to their location in the network. These initial value corresponds to their characteristics.

- According to our module calculate the total numbers of nodes associated to other node in the network, which means to have a information about which node is neighbor to other one. It even gives information about how many nodes are associated to each node.

- Now define the source and sink in the networks from calculated numbers of nodes to start the data transmission.

- A static data table with node distance, total no. of nodes associated to each and every node and bandwidth linked to each node etc will be obtained from observed data. The source node will now find the shortest path for communication which will be based on the data calculated. The node with least distance from source with highest bandwidth channel linked to it will be transmitted data from source.

- After receiving data from source this node will act as source and send data to other neighboring node with first priority to bandwidth and other to distance.

- At last whole coding function is given to a MATLAB, which is used for demonstration of whole processing. This module leads to less energy is consumption and even Failure of communication is reduced.

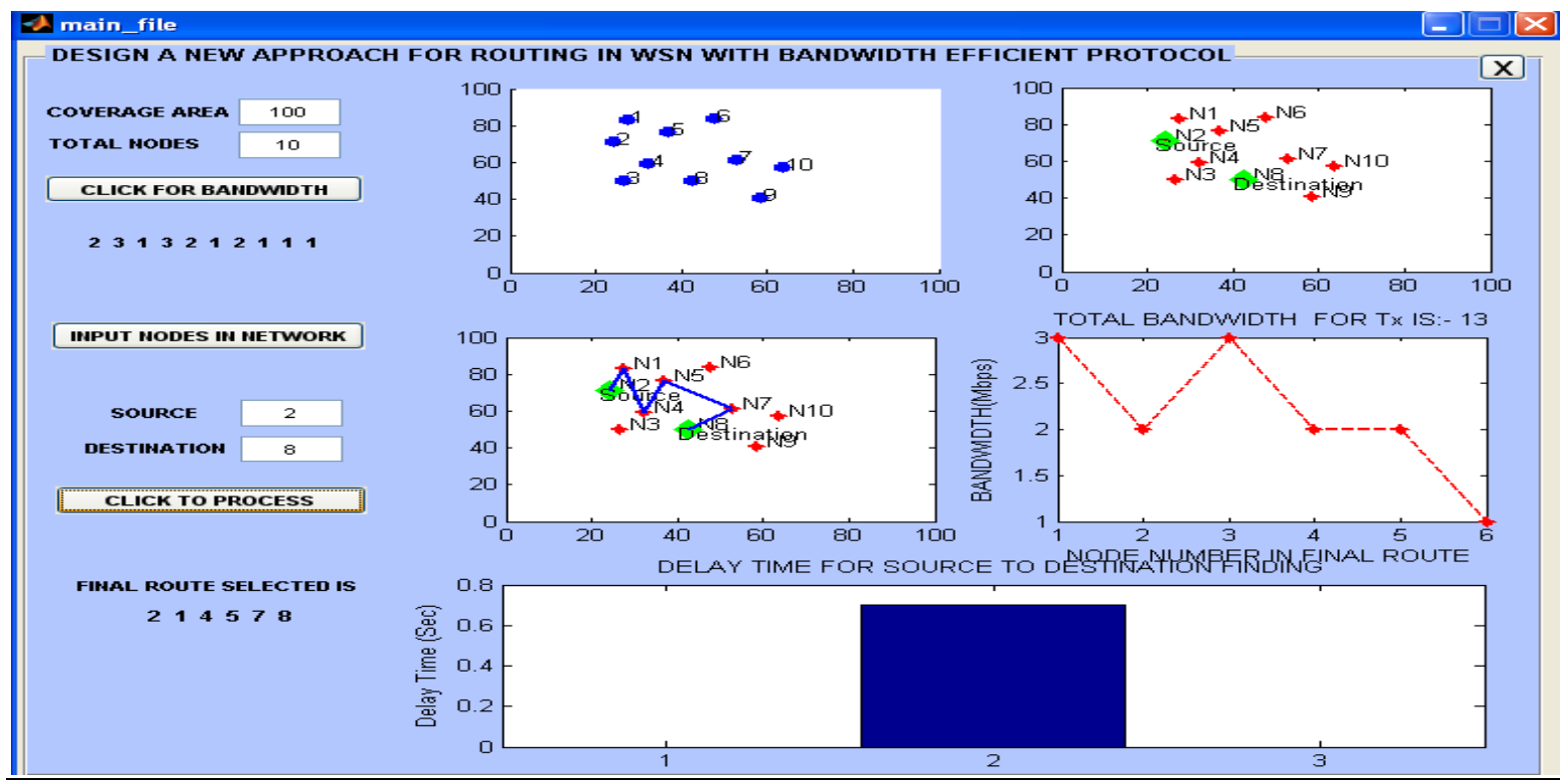

\section{CONCLUSION}

Advantages:-

- Delay Reduction

- Fast Data Transfer

- Maximum Throughput

- Efficient Routing

\section{Disadvantages:-}

If the proper bandwidth is not available for the routing then the whole networks works only on the basis of the distance. Hence, the delay time and cost of the network increases. If the distance is more than threshold then the network will not transfer the data. 


\section{REFERENCES}

[1] Amandeep and Gurmeet Kaur, "Performance Analysis of ADOV Routing Protocols in MANETs" International Journal of Engineering Science and Technology(IJEST) ISSN: 0975-5462, Vol.4 No. 08 August 2012, pp: $3620-3625$.

[2] A.K. Sharma and N. Bhatia, "Behavioral Study Of MANET Routing Protocols by using NS-2" International Journal Of Computation Engineering and Management, April 2011, Volume 12

[3] Mamoun Hussen Mamoun, "Location Aided Hybrid Routing Algorithm for MANET" in International Journal Of Engineering and Technology IJET/IJENS Vol. 11, No. 01, pp: 51-57, Feb 2011

[4] Resul Kara, Ibrahim Ozcelik and Huseyin Ekiz, "A new routing algorithm in MANETs: Position based hybrid routing" Scientific Research and Essays, 2010.

[5] Hossein Ashtiani, Shahpour Alirezaee, Seyed Mohsen Mir Hossein, Hamid Khoaravi, "PNR: New Position Based Routing Algorithm for Mobile Ad hoc Networks" Proceedings of the world congress on Engineering 2009 Vol. 1 WCE 2009, July 1 - 3, 2009 London, UK.

[6] Watanabe M, Higaki H., "NO-Beacon GEDIR: Location Based Ad - hoc Routing with less Communication Overhead" International Conference On Information Technology (ITNG 07), pp. 48 55, 2007.

[7] Demirci R (2007). Similarity relation matrix - based color edge detection. Int. j. Elect. Comm.(AEU) 61: $469-478$.

[8] Roger Watterhofer, "Algorithms for ad hoc and sensor networks", Computer Communications, 28 (2005) $1498-150$.

[9] Pallavi Kaura is a research scholar in Punjab Technical University and studing in DOABA Institute of Engineering and Technology, Mohali, Punjab, India.

[10] Miss Harshit is a Head Of Department in DOABA Institute of Engineering and Technology, Mohali, Punjab, India. 